\title{
Plasmon-driven surface catalysis in hybridized plasmonic gap modes
}

SUBJECT AREAS:

NANOPHOTONICS AND

PLASMONICS

NANOPARTICLES

Received

28 August 2014

Accepted

29 October 2014

Published

18 November 2014

Correspondence and requests for materials should be addressed to

Y.H. (yzhuang@cqu. edu.cn); S.W.

(wangshuxia@cqu.

edu.cn) or M.S.

(mtsun@iphy.ac.cn)

\author{
Hui Wang', Ting Liu', Yingzhou Huang', Yurui Fang ${ }^{2,3}$, Ruchuan Liu', Shuxia Wang', Weijia Wen ${ }^{1,4}$ \\ \& Mengtao Sun ${ }^{1,2}$
}

'Soft Matter and Interdisciplinary Research Center, College of Physics, Chongqing University, Chongqing, 400044, P. R. China, ${ }^{2}$ Beijing National Laboratory for Condensed Matter Physics, Institute of Physics, Chinese Academy of Sciences, Beijing, 100190, P. R. China, ${ }^{3}$ Division of Bionanophotonics, Department of Applied Phyiscs, Chalmers University of Technology, Göteborg, 41296, Sweden, ${ }^{4}$ Department of Phyiscs, The Hong Kong University of Science and Technology, Clear Water Bay, Kowloon, Hong Kong, China.

Plasmon-driven surface catalytic (PDSC) reaction in Ag/Au nanoparticle monomer or dimer-film gaps are experimentally and theoretically investigated, using surface enhanced Raman scattering (SERS) and finite element method. The variation of SERS spectra in different nano gaps of nanoparticle-film systems indicated the PDSC reaction was largely depended on the number of nanoparticles. The higher Raman intensity of $p, p^{\prime}$-dimercaptoazobenzene (DMAB) in dimer-film nanogap was because effective coupling of induced image charge on metal film in hybridized plasmonic gap mode, which was confirmed by the electric field distribution. Furthermore, the influence of material and wavelength was also studied to obtain the optimal experimental condition for best surface catalysis in hybridized plasmonic gap mode. Our studies in this common configuration of plasmonic nanostructure are of great significance not only in the field of catalysis on metal surface but also in other surface plasmon fields such as senor, photon detection, water splitting, etc.

M anipulating light under subwavelength presents an exciting reason to focus their attentions on surface plasmon in metal nanostructure by researchers all over the world ${ }^{1,2}$ The effective coupling of collective oscillation of free electrons and photons, surface plasmon polarizations (SPPs), confines and harvests the energy of light near the metal surface at nanoscale which decays exponentially along the normal direction. This highly confinement of electromagnetic (EM) field reaches the maximal and generates a quite huge enhanced EM field as large as several orders compared to incident light at metal surface, which are extensively applied in surface enhanced Raman scattering (SERS) ${ }^{3}$, localized surface plasmon sensor ${ }^{4}$, tip-enhanced Raman spectroscopy $^{5,6}$, nonlinear optics ${ }^{7,8}$, thermal image and therapy ${ }^{9}$, photo detection ${ }^{10,11}$, water splitting ${ }^{12,13}$, etc.

Besides these extensive application fields mention above, an interesting surface catalysis reaction driven by surface plasmon for its huge EM energy confined within subwavelength area attracted more and more attentions of researchers in the last several years ${ }^{14}$. This plasmonic driven surface catalysis (PDSC) reaction was discovered through three enhanced SERS peaks of $p$-aminothiophenol (PATP) molecule absorbed on metal surface. The strong enhanced Raman peaks at 1143,1390 and $1432 \mathrm{~cm}^{-1}$ of PATP were explained by chemical enhancement of SERS since $1994^{15}$. However, in $2010 \mathrm{Wu}$ and co-worker predicted theoretically that a PDSC reaction that $p, p^{\prime}-$ dimercaptoazobenzene (DMAB) could be produced from $p$-aminothiophenol (PATP) on Ag nanoparticles and those three enhanced Raman peaks were the Raman feature of DMAB molecule ${ }^{16}$. This predication was immediately demonstrated by experiments and further confirmed by a series reports in various experiment conditions and theoretical methods $s^{17-27}$.

Since the surface plasmon varies a lot in different metal nanostructures ${ }^{2}$, various metal surfaces were investigated to verify the PDSC reaction, such as colloids ${ }^{17}$, nanowires ${ }^{28}$, film ${ }^{22,29}$, tips ${ }^{19,25}$, single particle ${ }^{30}$, nanoparticles dimer ${ }^{31,32}$, etc. Among them, the nanoparticle-film systems were extensively studied in various plasmonic fields, not only for its simple configuration and convenient fabrication but also for its highly confined EM field in nanogaps between nanoparticle and film $\mathrm{m}^{22,30,33}$. Compared to common metal colloids SERS system, nanogaps in nanoparticle-film were more uniform and could realize selective enhancement in a subwavelength area on film ${ }^{34}$. Since the plasmonic properties in nanogap come from the hybridization between surface plasmon of nanoparticle and that of metal film, the size, shape of nanoparticle and the thickness of film has a great effect on the enhancement in nanogap. Interesting, we recently found the EM distribution of this hybridized plasmonic gap mode was also largely influenced by the number of nanopar- 
ticles that the dimer-film nanogap had an unusual greater capability to confine light energy than monomer-film nanogap ${ }^{30}$. In this year, Yang, Ren and co-workers had confirmed our results and indicated this hybridized plasmon gap mode could be modulated by the wavelength of incident light ${ }^{35}$. Therefore, the PDSC reaction in this hybridized plasmon gap mode could be an interesting topic to study the catalysis on metal surface.

In this work, the PDSC reactions in Au/Ag nanoparticle-film gaps were investigated through SERS and finite element method. The SERS spectra of PATP excited by $633 \mathrm{~nm}$ laser or $532 \mathrm{~nm}$ laser in $\mathrm{Au} / \mathrm{Ag}$ nanoparticle monomer or dimer-film gaps were measured. The results figured out the numbers of nanoparticle were quite important in this surface catalysis. To understand this phenomenon, the distribution and the enhancement of electric field in the nanoparticle-film gaps were analyzed through finite element method. Considering the Ag:Au bimetallic nanoparticle could exhibit different plasmonic properties, the SERS spectra of Au and Ag mixed nanoparticle dimer-film system were also investigated and the results confirmed the PDSC reaction occurred in $\mathrm{Au}$ or Ag pure metal dimer-film system. Since the simple configuration and extensive application of nanoparticle-film system in plasmonics, our work in nanoparticle-film gaps could be an important and interesting supplement in PDSC field.

\section{Results}

Raman spectra of PATP excited by $633 \mathrm{~nm}$ laser. The SERS spectra of PATP in nanoparticle-film gaps excited by $633 \mathrm{~nm}$ laser were first investigated as shown in Figure 1 and all the nanoparticle monomer and its corresponding nanoparticle dimer were located on the same film in our work, and then the influence of background light had been eliminated.

The SERS spectra in Figure 1(a) were obtained in gaps between Ag nanoparticle monomer or dimer and Ag film whose SEM image were inset. The scale bar in SEM image indicated the nanoparticle from either Ag monomer or dimer has similar radii of about $50 \mathrm{~nm}$. And the obvious Raman feature of DMAB in Figure 1(a) demonstrated PDSC were performed in both monomer-film and dimer-film gaps. Furthermore, the larger than twice stronger Raman intensity in spectra figured out greater electric field generated in dimer-film gap compared to monomer-film gap.

The experiment data of Au nanoparticle monomer or dimer on $\mathrm{Ag}$ film were present in Figure 1(b). The much stronger Raman intensity
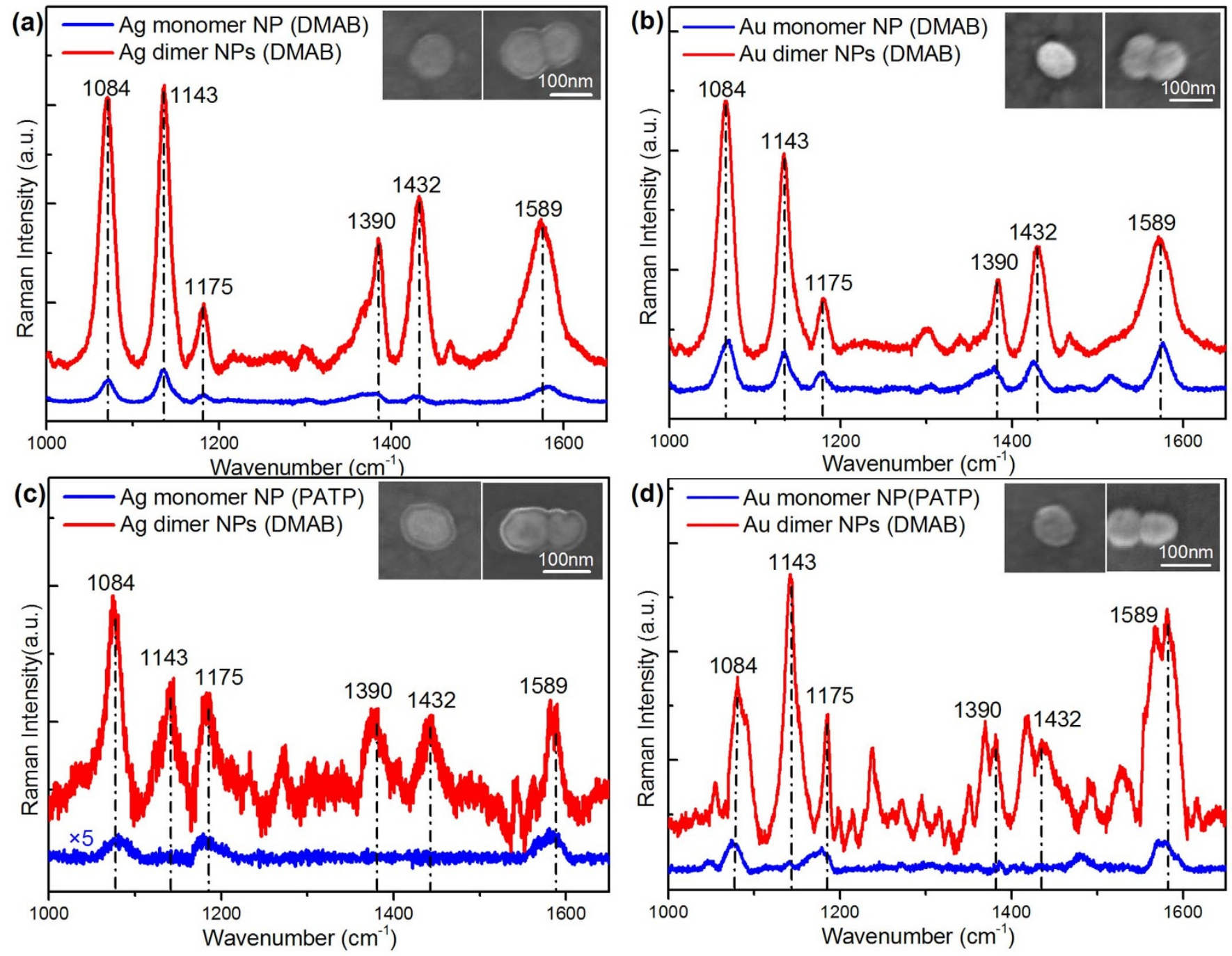

Figure 1 SERS spectra of PATP absorbed on Ag/Au films excited by $633 \mathrm{~nm}$ laser. (a) in Ag nanoparticle monomer-Ag film gaps (blue line) or Ag nanoparticle dimer-Ag film (red line) gaps, (b) in Au nanoparticle monomer-Ag film gaps (blue line) or Au nanoparticle dimer-Ag film gaps (red line), (c) in Ag nanoparticle monomer-Au film gaps (blue line) or Ag nanoparticle dimer-Au film gaps (red line), (d) in Au nanoparticle monomer-Au film gaps (blue line) or Au nanoparticle dimer-Au film gaps (red line). Insets are the corresponding SEM image of Ag/Au nanoparticle-film. 
of DMAB in spectra presented the similar conclusion while the radii of all Au nanoparticles were about $40 \mathrm{~nm}$ as shown in the inset SEM image. All results above indicated PDSC were performed on Ag film in our experiment and higher electric field was generated in gaps of dimer-film for the much stronger Raman intensity.

The SERS spectra in Figure 1(c) were collected in gaps between Ag nanoparticle monomer or dimer and Au film whose corresponded inset SEM image illustrated the diameters of all Ag nanoparticles were about $100 \mathrm{~nm}$. To make Raman feature clear in figure, the weak SERS spectra from monomer-film gap was 5 times amplified. The additional Raman peak at 1143, 1390 and $1432 \mathrm{~cm}^{-1}$, related to $\mathrm{N}=\mathrm{N}$ of $\mathrm{DMAB}$ vibrational modes, turned up in spectra from dimer-film gaps presented an obvious different Raman feature compared to the other one. This result pointed out that the PDSC were performed in the dimer-film gap while it did not occur in the monomer-film gap, in which the SERS spectra presented Raman feature of PATP. Similar conclusion was obtained in Au nanoparticles-Au film system by the SERS spectra and their corresponding inset SEM images in Figure 1(d). With similar diameter of about $80 \mathrm{~nm}$, the Raman feature of DMAB only turned up in the nanoparticle dimerfilm gaps figured out the PDSC was not performed in the nanoparticle monomer -Au film gap.
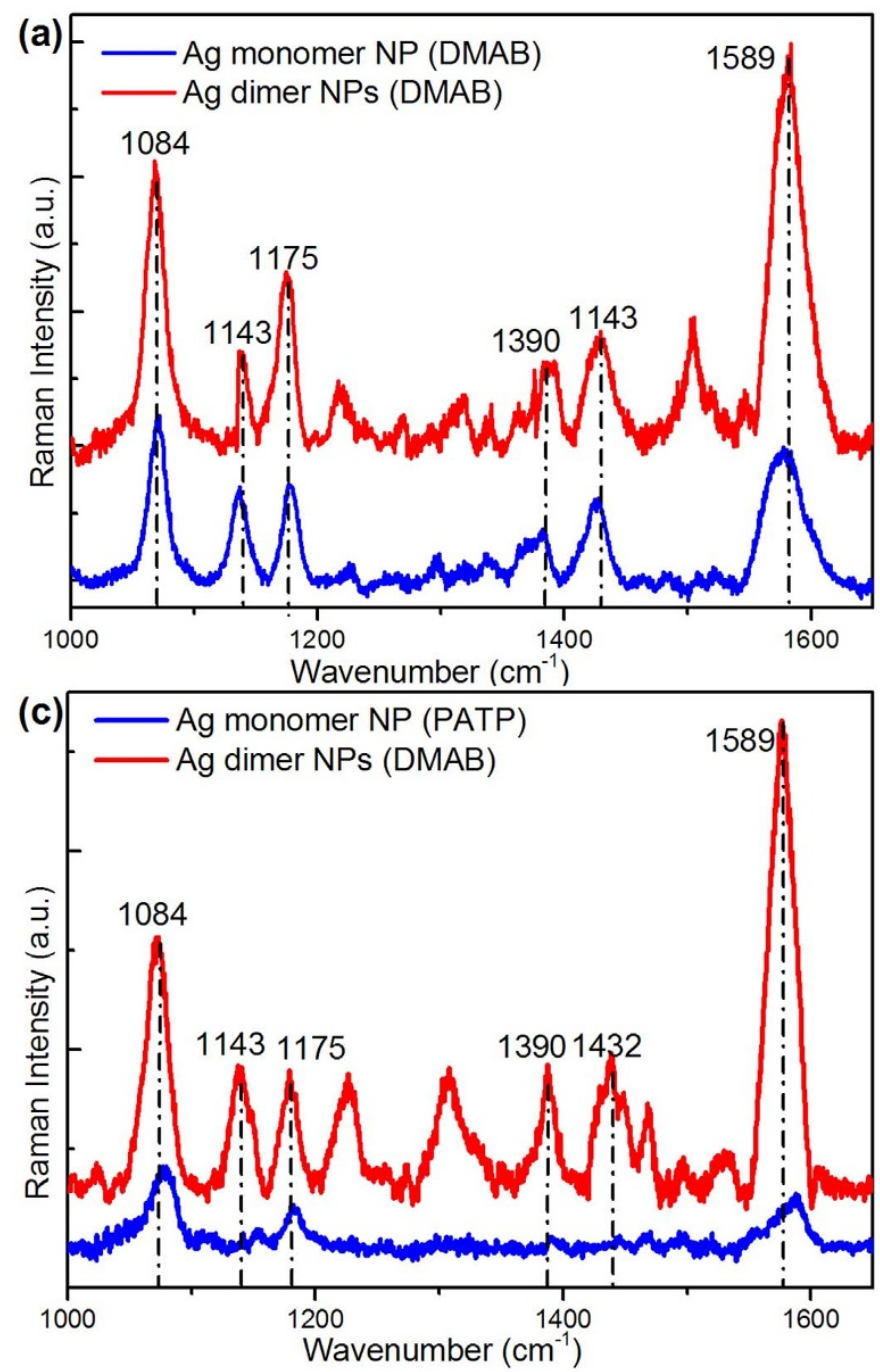

SERS spectra of PATP excited by $532 \mathrm{~nm}$ laser. To demonstrate the conclusion discussed above under different exciting laser, the SERS in the same systems excited by $532 \mathrm{~nm}$ laser were also measured in Figure 2. The similar phenomena were observed in all nanoparticleAg film systems that the PDSC were performed while the higher Raman intensity of DMAB were collected in dimer-film gap as illustrated in Figure 2(a) and 2(b). Due to the strong interband electronic transitions of gold irradiated by $532 \mathrm{~nm}$ light, there were much difference in nanoparticle-Au film system such as the appearance of intensive fluorescence background without identifiable Raman feature in Figure 2(d). However, the existence of Ag nanoparticle reserved the Raman signal of molecule on Au film shown in Figure 2(c) which guided energy of light into localized surface plasmons (LSPs) at Ag nanoparticle surface but not interband electronic transition in Au film. The SERS spectra in Figure 2(c) were consistent with the result in Figure 1(c) that the PDSC only took place in the Ag nanoparticle dimer-Au film gaps.

The electric field in Au/Ag nanoparticle-film gap systems excited by $633 \mathrm{~nm}$ laser. Through finite element method, Figure 3 illustrated the distribution and enhancement of electric field in nanoparticle and film systems, which provide a useful way to
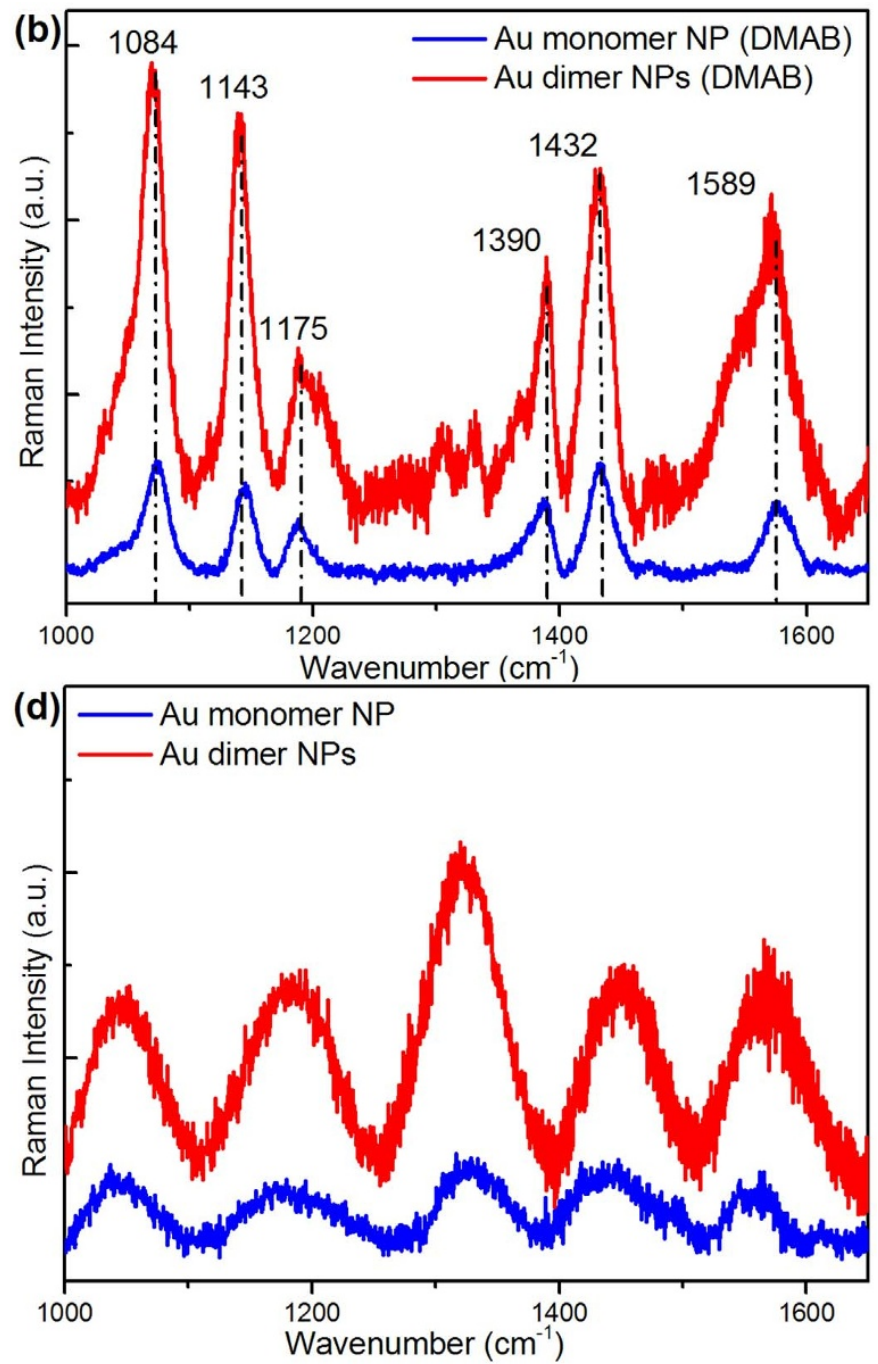

Figure $2 \mid$ SERS spectra of PATP absorbed on Ag/Au films excited by 532 nm laser. (a) in Ag nanoparticle monomer-Ag film gaps (blue line) or Ag nanoparticle dimer-Ag film (red line) gaps, (b) in Au nanoparticle monomer-Ag film gaps (blue line) or Au nanoparticle dimer-Ag film gaps (red line), (c) in Ag nanoparticle monomer-Au film gaps (blue line) or Ag nanoparticle dimer-Au film gaps (red line), (d) in Au nanoparticle monomer-Au film gaps (blue line) or Au nanoparticle dimer-Au film gaps (red line). 

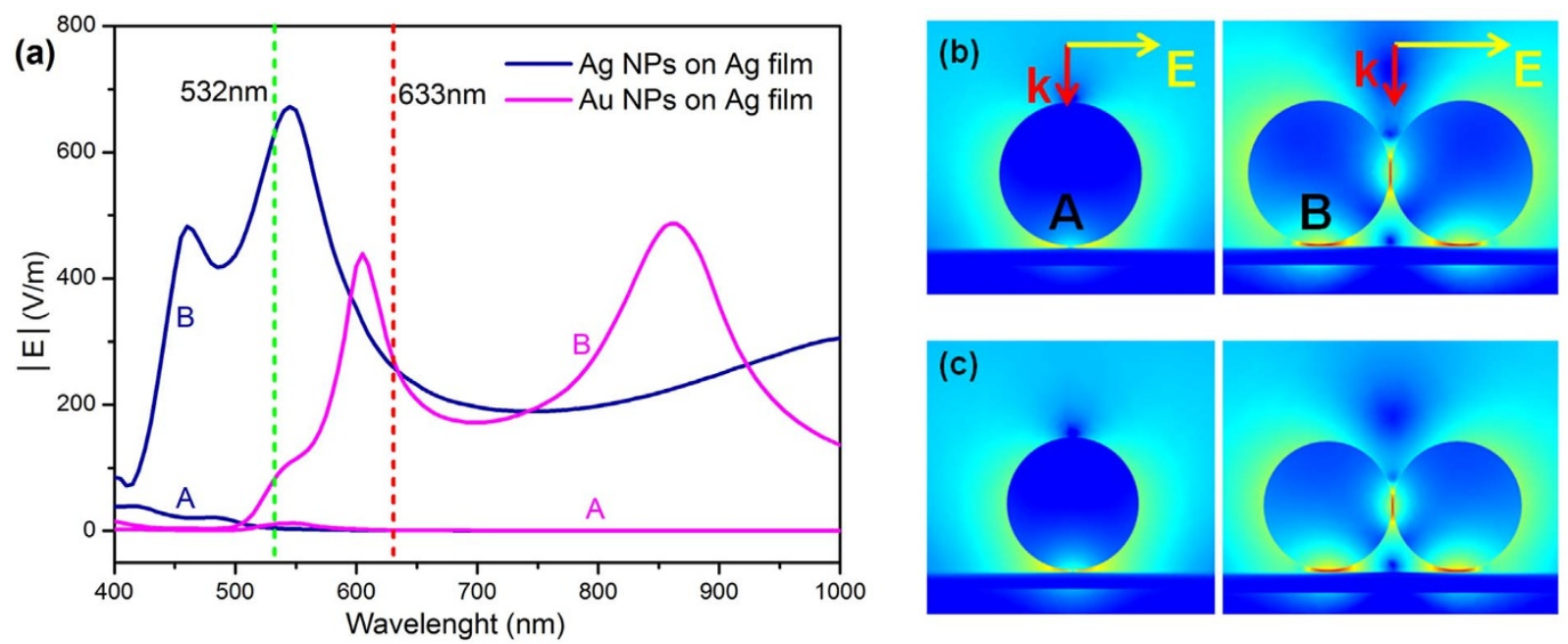

Log|티
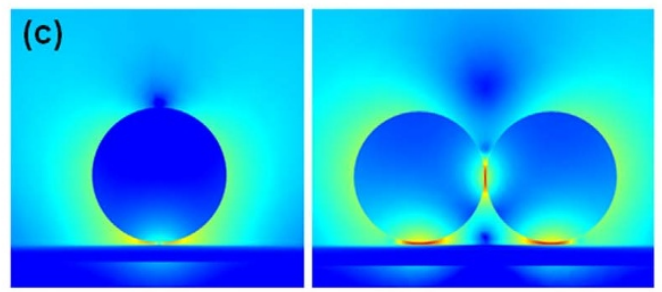

4.8917
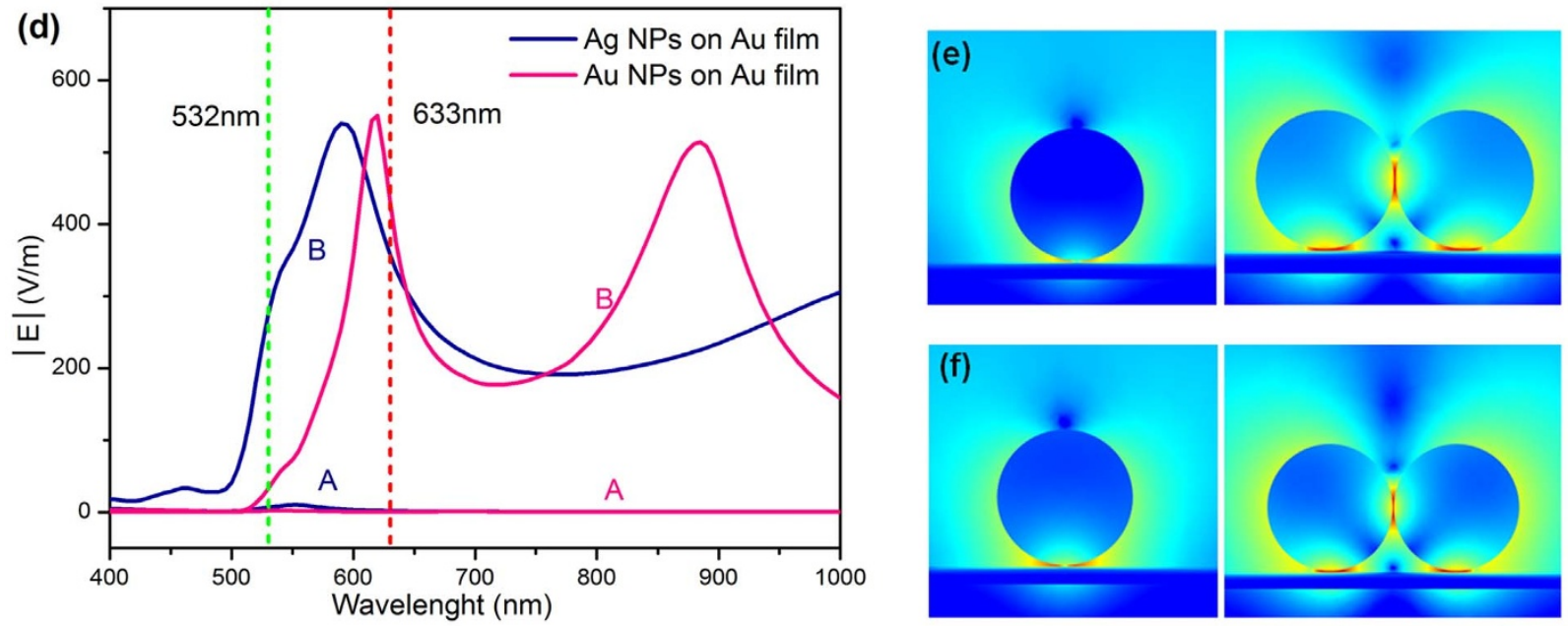

4

2

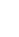

Figure $3 \mid$ The distribution and enhancement of electric field in nanoparticle and film systems excited by $633 \mathrm{~nm}$ laser. (a) E field enhancement in gaps between Ag/Au nanoparticle and Ag film. Gap A represent gap between nanoparticle monomer and film while Gap B represent gap between nanoparticle dimer and film. (b) E field distribution in the gaps between Ag nanoparticles monomer or dimer-Ag film. (c) E field distribution in gaps between Au nanoparticles monomer or dimer-Ag film. (d) E field enhancement in gaps between Ag/Au nanoparticle and Au film. (e) E field distribution in gaps between Ag nanoparticles monomer or dimer-Au film. (f) E field distribution in gaps between Au nanoparticles monomer or dimer-Au film. The $633 \mathrm{~nm}$ laser is the light source in all E field distribution here.

analyze the LSP in this hybridized plasmonic gap mode. The images in Figure 3 clearly indicated the light energy was highly focused at the gaps in the system, which was largely influenced by the number of nanoparticle on metal film. For example in Ag nanoparticle monomer or dimer-Ag film systems, the deeper color with smaller area in Figure 3(b) pointed out the higher energy density of LSP generated in dimer-film gap excited by $633 \mathrm{~nm}$ laser. The electric enhancement as a function of incident light wavelength in Figure 3(a) provided the detail data of LSP energy density difference in monomer-film and dimer-film gap. Here gap A represented the gap between nanoparticle monomer and film while gap B represented the gap between nanoparticle dimer and film in this work. The green and red dash line in Figure 3(a) represented the wavelength of exciting laser in our experiment above, $532 \mathrm{~nm}$ and $633 \mathrm{~nm}$. It was quite clear that the intensity of electric field in dimerfilm gap was much stronger than that in monomer-film gap in whole visible region. This higher energy density in dimer-film gap was also demonstrated in nanoparticle-Au film by the images of Figure 3(df), which was responsible to the selective catalysis on smoother surface of $\mathrm{Au}$ film in our experiments. Because of the inevitable roughness of metal nanostructure caused by fabrication process, the difference of simulated electric field in two kinds of gaps was much greater compared to the experiment SERS data.
SERS spectra and electric field of Au and Ag mixed dimer-film system excited by $633 \mathrm{~nm}$ laser. Since the Ag:Au bimetallic nanoparticle showed different plasmonic properties compared to pure $\mathrm{Ag}$ or $\mathrm{Au}$ nanoparticle ${ }^{36}$, the PDSC reaction was also investigated in $\mathrm{Au}$ and $\mathrm{Ag}$ mixed dimer-film system, whose SERS spectra and electric field were illustrated in Figure 4. To distinguish the material of nanoparticle dimer in film, the Energy dispersive spectroscopy (EDS) equipped on SEM was used to identify the $\mathrm{Ag}$ or Au nanoparticle, whose data was presented in supplementary information. The Raman feature peak of DMAB in Figure 4(a) and (b) indicated the PDSC did occur in mixed dimer-film system as the pure dimer-film system, either on Ag film or on Au film. The results were confirmed by the electric distribution in Figure 4(c) and (d) that the light energy was highly confined in the dimer-particle gaps.

\section{Discussion}

Since both nanoparticle monomer and its corresponding dimer in our experiments were located on the same metal film, the distributions of PATP molecule in all nanoparticle-film gaps were extremely similar. Therefore, the difference of SERS intensities and selective catalysis discussed above could be understood by variation of light 

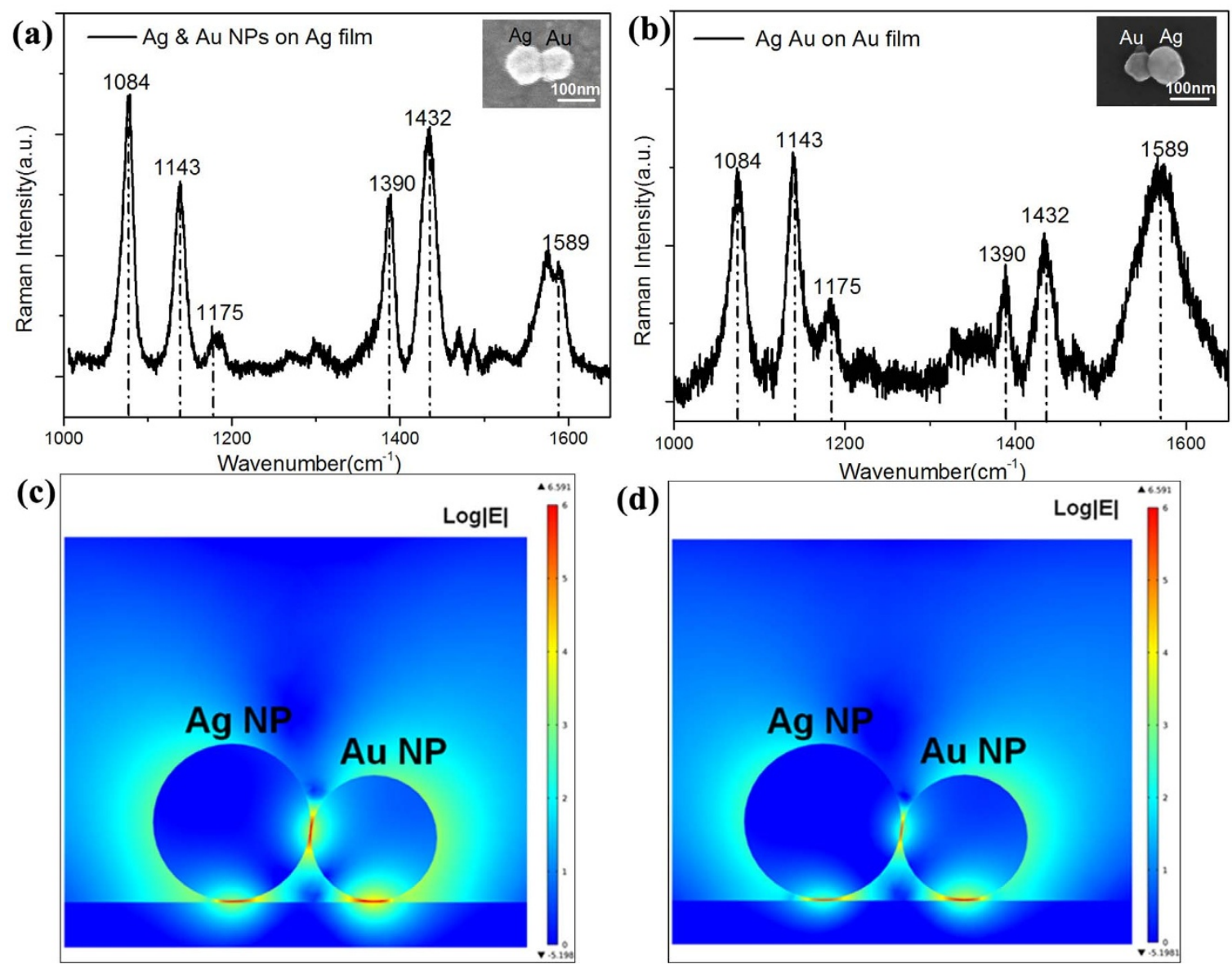

(d)

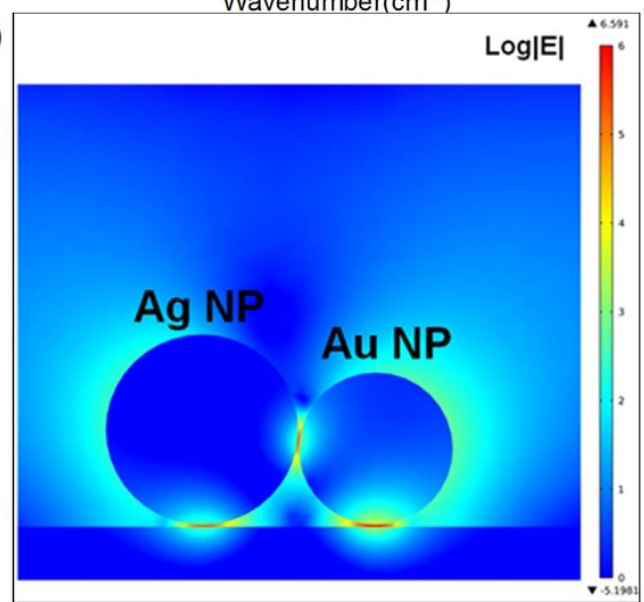

Figure 4 SERS spectra and electric field of Au mixing with Ag nanoparticle-film system excited by $633 \mathrm{~nm}$ laser. (a) in Au mixing Ag nanoparticle-Ag film system, (b) in Au mixing Ag nanoparticle-Au film system. (c) E field distribution in gaps between Au mixing Ag nanoparticle and Ag film. (d) E field distribution in gaps between Au mixing Ag nanoparticle and Au film. Insets are the corresponding SEM of Au mixing with Ag nanoparticle-film.

energy in nanoparticle-film gaps, which only came from the configuration of the metal nanostructure.

Obviously, the number of nanoparticles on film dominated the result that SERS intensities of dimer-film system were much stronger than that in monomer-film system, either on $\mathrm{Ag}$ or $\mathrm{Au}$ film. Furthermore, the larger than twice stronger Raman intensity in each dimer-film gap compared to monomer-film gap meant much more light energy confined in dimer-film gaps to drive surface catalysis. This phenomenon could be analyzed by the hybridization between surface plasmons of metal nanoparticle and that of metal film. In monomer-film system, the image charge on metal surface induced by surface charge on single nanoparticle centralized at two side of nanoparticle-film gap, which could not effectively confine light energy in the gap. While in the dimer-film situation, the strong coupling of nanoparticle dimer made the induced image charge on metal film just located at the two dimer-film gaps. The effective coupling gave rise to the highly focused light in gaps which brought out the strong Raman intensity and PDSC reactions. Detail demonstration could be seen in our recent work ${ }^{30}$.

As materials of nanostructures played an important role in surface plasmon properties, our results also illustrated the difference in PDSC reactions of $\mathrm{Ag}$ or $\mathrm{Au}$ system. In comparing parts (a) and (b) of Figure 1, it is obviously that all Raman spectra were quiet similar and appeared Raman feature of DMAB on Ag film, which meant the PDSC reactions occurred accordingly. However, when the Ag nanoparticles were located on different substrate in Figure 1(a) and (c), the obvious difference in SERS spectra demonstrated the influence of material substrate was great. The Raman peaks at 1143,1390 and $1432 \mathrm{~cm}^{-1}$ indicated the light energy confined by the hybridized plasmonic gap mode in $\mathrm{Ag}$ nanoparticle dimer-Ag film gap was strong enough to undergo the PDSC reaction. While in $\mathrm{Ag}$ nanoparticle monomer-Au film situation, the SERS spectra illustrated the PDSC reaction could not smoothly occur for weaker light energy in gaps. These experiment results were different from our simulation results in Figure 3, in which the enhancements in $\mathrm{Ag}$ monomer-Ag film gap or -Au film gap were almost the same. This was mainly attributed to the rougher surface of $\mathrm{Ag}$ film caused by fabrication technology that the roughness of $\mathrm{Ag}$ and $\mathrm{Au}$ film were 3.25 and $2.249 \mathrm{~nm}$, respectively (the SERS spectra of Ag or Au film without nanoparticle was present in supplementary information).

The three DMAB Raman peaks in SERS spectra of Figure 4 demonstrated $\mathrm{Ag}$ and $\mathrm{Ag}$ mixed nanoparticle dimer had similar ability to drive surface catalysis on $\mathrm{Ag}$ or Au film as the pure nanoparticle dimer. The fact was confirmed by the electric distribution in Figure 4(c) and (d) that the electric field was greatly enhanced in the dimer-film gaps no matter what material consisted in the hybridized plasmonic gap mode. This was because the Ag has similar permittivity as Au in the visible range that the hybridization between surface plasmon of noble metal nanoparticle dimer and noble metal film were almost the same. These results indicated the number of nanoparticle in PDSC reaction of hybridized plasmonic gap mode played an much more important role than material.

In summary, the PDSC reaction of DMAB produced from PATP in $\mathrm{Au} / \mathrm{Ag}$ nanoparticle-film gaps were examined by SERS. The stron- 
ger SERS intensity of DMAB or selective catalysis in dimer-film gaps indicated much more light energy was confined in the dimer-film gaps for the hybridization between surface plasmon of metal nanoparticle and metal film, which was confined by the electric field distribution through finite element method. All results demonstrated the particle's number played a core role in the hybridized plasmonic gap mode for the coupling efficiency of induced image charge on metal film. Due to the simple configuration and convenient fabrication of nanoparticle-film systems, our result was quite useful not only in application of catalysis on metal surface, but also in other surface plasmon fields such as sensor, photon detection, water splitting and etc.

\section{Methods}

PATP was purchased from Aladdin Industrial Corporation. The substrate for SERS measurement was prepared by evaporating Ag and Au metal onto silicon under a high vacuum using the electron beam evaporation system (model Peva-600E). The evaporation conditions were controlled to produce a layer of $\mathrm{Ag}$ and $\mathrm{Au}$ all with an average thickness of $100 \mathrm{~nm}$.

The surface roughness was evaluated with atomic force microscopy (AFM), the roughness of $\mathrm{Ag}$ and $\mathrm{Au}$ film were 3.25 and $2.249 \mathrm{~nm}$, respectively.

The element of mixed dimer on metal film was distinguished by Energy dispersive spectroscopy (EDS).

The Ag and Au nanoparticles were synthesized by polyol method according to references. The average diameters were $100 \mathrm{~nm}$ and $80 \mathrm{~nm}$ for Ag and Au nanoparticle, respectively. To make sure the molecule only located in the gaps between nanoparticle and film, the $\mathrm{Ag}$ and $\mathrm{Au}$ films were firstly immerged in a $1^{*} 10^{-5} \mathrm{M}$ ethanol solution of PATP for more than $2 \mathrm{~h}$. And then the films were washed with ethanol and deionized water for $3 \mathrm{~min}$ and dried with $\mathrm{N}_{2}$ gas. At last the $\mathrm{Ag} / \mathrm{Au}$ nanoparticle monomer and dimer were fabricated by spin-coating $\mathrm{Ag} / \mathrm{Au}$ nanoparticle solution spin coating on the $\mathrm{Ag} / \mathrm{Au}$ film.

All the SRES measurements in this work were performed by a commercial MicroRaman spectrometer (Horibba) with a $633 \mathrm{~nm}$ or $532 \mathrm{~nm}$ laser.

The near-field distribution of the electric (E) fields was calculated using the finite element method (COMSOL 4.3a commercial package). The system of monomer or dimer Ag/Au nanoparticles located $1 \mathrm{~nm}$ above the $\mathrm{Ag} / \mathrm{Au}$ films ( $100 \mathrm{~nm}$ thick), with a $1 \mathrm{~nm}$ edge-to-edge dimer separation. The illumination was from the particle side, normal to the substrate, with a polarization along the dimer axis and an E field component is $1 \mathrm{~V} / \mathrm{m}$. The excited wavelengths are $633 \mathrm{~nm}$.

1. Ozbay, E. Plasmonics: Merging Photonics and Electronics at Nanoscale Dimensions. Science 311, 189-193 (2006).

2. Halas, N. J., Lal, S., Chang, W. S., Link, S. \& Nordlander, P. Plasmons in Strongly Coupled Metallic Nanostructures. Chem. Rev. 111, 3913-3961 (2011).

3. Fan, X., Zheng, W. \& Singh, D. J. Light scattering and surface plasmons on small spherical particles. Light: Sci. Appl. 3, e179, (2014). Doi:10.1038/lsa.2014.60.

4. Mayer, K. M. \& Hafner, J. H. Localized Surface Plasmon Resonance Sensors. Chem. Rev. 111, 3828-3857 (2011).

5. Sun, M., Zhang, Z., Chen, L., Sheng, S. \& Xu, H. Plasmonic Gradient Effects on High Vacuum Tip-Enhanced Raman Spectroscopy. Adv. Optical Mater. 2, 74-80 (2014).

6. Sun, M., Zhang, Z., Kim, Z. H., Zheng, H. \& Xu, H. Plasmonic scissors for molecular design. Chem. Eur. J. 19, 14958-14962 (2013).

7. Oulton, R. F. et al. Plasmon Lasers at Deep Subwavelength Scale. Nature 461, 629-632 (2009).

8. Zhang, Y., Wen, F., Zhen, Y. R., Nordlander, P. \& Halas, N. J. Coherent Fano Resonances in a Plasmonic NanoclusterEenhance Optical Four-Wave Mixing. PNAS. 110, 9215-9219 (2013).

9. Loo, C., Lowery, A., Halas, N., West, J. \& Drezek, R. Immunotargeted Nanoshells for Integrated Cancer Imaging and Therapy. Nano Lett. 5, 709-711 (2005).

10. Chalabi, H., Schoen, D. \& Brongersma, M. L. Hot-Electron Photodetection with a Plasmonic Nanostripe Antenna. Nano Lett. 14, 1374-1380 (2014).

11. Knight, M. W., Sobhani, H., Nordlander, P. \& Halas, N. J. Photodetection with Active Optical Antennas. Science 332, 702-704 (2011).

12. Lee, J., Mubeen, S., Ji, X., Stucky, G. D. \& Moskovits, M. Plasmonic Photoanodes for Solar water Splitting with Visible Light. Nano Lett. 12, 5014-5019 (2012).

13. Mubeen, S. et al. An Autonomous Photosynthetic Device in Which All Charge Carriers Derive from Surface Plasmons. Nature Nanotech. 8, 247-251 (2013).

14. Sun, M. \& Xu, H. A Novel Application of Plasmonics: Plasmon-Driven SurfaceCatalyzed Reactions. Small 8, 2777-2786 (2012).

15. Osawa, M., Matsuda, N., Yoshii, K. \& Uchida, I. Charge Transfer Resonance Raman Process in Surface-Enhanced Raman Scattering from $\mathrm{p}$-Aminothiophenol Adsorbed on Silver: Herzberg-Teller Contribution. J. Phys. Chem. 98, 12702-12707 (1994).

16. $\mathrm{Wu}, \mathrm{D}$. Y. et al. Photon-driven charge transfer and photocatalysis of $\mathrm{p}$ aminothiophenol in metal nanogaps: a DFT study of SERS. Chem. Commun. 47, $2520-2522$ (2011).
17. Fang, Y., Li, Y., Xu, H. \& Sun, M. Ascertaining p,p'-Dimercaptoazobenzene Produced from p-Aminothiophenol by Selective Catalytic Coupling Reaction on Silver Nanoparticles. Langmuir 26, 7737-7746 (2010).

18. Huang, Y. F. et al. When the Signal Is Not from the Original Molecule To Be Detected: Chemical Transformation of para-Aminothiophenol on Ag during the SERS Measurement. J. Am. Chem. Soc. 132, 9244-9246 (2010).

19. Sun, M., Zhang, Z., Zheng, H. \& Xu, H. In-Situ Plasmon-Driven Chemical Reactions Revealed by High Vacuum Tip-Enhanced Raman Spectroscopy. Sci. Rep. 2, 647 (2012).

20. Kang, L. et al. Amino Acid-Assisted Synthesis of Hierarchical Silver Microspheres for Single Particle Surface-Enhanced Raman Spectroscopy. J. Phys. Chem. C 117, 10007-10012 (2013).

21. Zhang, Z. et al. Insights into the Nature of Plasmon-Driven Catalytic Reactions Revealed by HV-TERS. Nanoscale 5, 3249-3252 (2013).

22. Huang, Y., Fang, Y., Yang, Z. \& Sun, M. Can p,p'-Dimercaptoazobisbenzene Be Produced from p-Aminothiophenol by Surface Photochemistry Reaction in the Junctions of a Ag Nanoparticle-Molecule-Ag (or Au) Film. J. Phys. Chem. C 114, 18263-18269 (2010).

23. Sun, M., Huang, Y., Xia, L., Chen, X. \& Xu, H. The pH-Controlled PlasmonAssisted Surface Photocatalysis Reaction of 4-Aminothiophenol top, $\mathrm{p}^{\prime}$ Dimercaptoazobenzene on Au, Ag, and Cu Colloids. J. Phys. Chem. C 115, 9629-9636 (2011).

24. Huang, Y. \& Dong, B. PH Dependent Plasmon-Driven Surface-Catalysis Reactions of $\mathrm{p}, \mathrm{p}^{\prime}$-Dimercaptoazobenzene Produced from para-Aminothiophenol and 4-Nitrobenzenethiol. Sci China Chem. 55, 2567-2572 (2012).

25. Zhang, X., Wang, P., Zhang, Z., Fang, Y. \& Sun, M. Plasmon-driven sequential chemical reactions in an aqueous environment. Sci. Rep. 4, 5407 (2014).

26. Xu, P. et al. Mechanistic understanding of surface plasmon assisted catalysis on a single particle: cyclic redox of 4-aminothiophenol. Sci. Rep. 3, 2997 (2013).

27. Huang, Y., Fang, Y., Zhang, Z., Zhu, L. \& Sun, M. Nanowire-supported plasmonic waveguide for remote excitation of surface-enhanced Raman scattering. Light: Sci. Appl. 3, e199, (2014). Doi:10.1038/lsa.2014.80.

28. Fang, Y., Wei, H., Hao, F., Nordlander, P. \& Xu, H. Remote-excitation surfaceenhanced Raman scattering using propagating Ag nanowire plasmons. Nano Lett. 9, 2049-2053 (2009).

29. Dong, B., Fang, Y., Chen, X., Xu, H. \& Sun, M. Substrate-, wavelength-, and timedependent plasmon-assisted surface catalysis reaction of 4-nitrobenzenethiol dimerizing to $\mathrm{p}, \mathrm{p}^{\prime}$-dimercaptoazobenzene on $\mathrm{Au}, \mathrm{Ag}$, and $\mathrm{Cu}$ films. Langmuir 27, 10677-10682 (2011).

30. Fang, Y. \& Huang, Y. Electromagnetic Field Redistribution in Hybridized Plasmonic Particle-Film System. Appl. Phys. Lett. 102, 153108 (2013).

31. Shegai, T. et al. A bimetallic nanoantenna for directional colour routing. Nat. Commun. 2, 481-485 (2011).

32. Toroghia, S., Lumdeea, C. \& Kik, P. G. Cascaded plasmon resonances multimaterial nanoparticle trimers for extreme field enhancement. Proc. of SPIE. 8809, 88091M (2013).

33. Li, J. F. et al. Shell-Isolated Nanoparticle-Enhanced Raman Spectroscopy. Nature 464, 392-395 (2010).

34. Tanaka, Y., Sanada, A. \& Sasaki, K. Nanoscale interference patterns of gap-mode multipolar plasmonic fields. Sci. Rep. 2, 764 (2012).

35. Wang, X. et al. Probing the Location of Hot Spots by Surface-Enhanced Raman Spectroscopy: Toward Uniform Substrates. ACS Nano. 8, 528-536 (2014).

36. Fan, M. et al. Surface-enhanced Raman Scattering (SERS) from Au:Ag bimetallic nanoparticles: the effect of the molecular probe. Chem. Sci. 4, 509-515 (2013).

\section{Acknowledgments}

This work was supported by the National Natural Science Foundation of China (Grant Nos. $11204390,91436102,11374353,11474141$ ), Natural Science Foundation Project of CQ CSTC 2014jcyjA40002, Fundamental Research Funds for the Central Universities CQDXWL-2013-009, the Program of Liaoning Key Laboratory of Semiconductor Light Emitting and Photocatalytic Materials, the Program of Shenyang Key Laboratory of Optoelectronic Materials and Technology (F12-254-1-00) and Special Fund for Agro-scientific Research in the Public Interest (NO. 201303045).

\section{Author contributions}

Y.H. and M.S. supervised the project. Y.H. and M.S. designed the experiments. H.W. and T.L. experimentally measured the SERS spectra. R.L. experimentally measured the roughness of metal film, Y.F. and W.W. did simulation. Y.H. and M.S. analysed the data Y.H. and M.S. wrote the paper, and Y.H., S.W. and M.S. discussed and revised the manuscript.

\section{Additional information}

Supplementary information accompanies this paper at http://www.nature.com/ scientificreports

Competing financial interests: The authors declare no competing financial interests.

How to cite this article: Wang, H. et al. Plasmon-driven surface catalysis in hybridized plasmonic gap modes. Sci. Rep. 4, 7087; DOI:10.1038/srep07087 (2014). 

NoDerivs 4.0 International License. The images or other third party material in this article are included in the article's Creative Commons license, unless indicated otherwise in the credit line; if the material is not included under the Creative
Commons license, users will need to obtain permission from the license holder in order to reproduce the material. To view a copy of this license, visit http:// creativecommons.org/licenses/by-nc-nd/4.0/ 